\title{
DATA FUSION FOR DRAWING AND ANALYSIS OF AN ANCIENT ROMAN BOAT IN HERCULANEUM
}

\author{
A. Bosco ${ }^{1}$, E. Canna ${ }^{2}$, L. Carpentiero ${ }^{1}$, A. D’Andrea ${ }^{1}$, F. Forte $^{1}$, E. Minucci ${ }^{1}$, R. Valentini ${ }^{1}$, C. Zazzaro ${ }^{1}$ \\ ${ }^{1}$ Università degli Studi di Napoli “L’Orientale” - abosco@unior.it, lauracarpentiero@yahoo.it, dandrea@unior.it, \\ francescaforte88@gmail.com, eleonoraminucci1@gmail.com,rvalentini@unior.it,czazzaro@unior.it \\ ${ }^{2}$ Archaeological Park of Herculaneum - elisabetta.canna-01@beniculturali.it
}

Commission II

KEY WORDS: low-cost 3D technologies, Laser Scanner, Photogrammetry, Herculaneum, Boat

\begin{abstract}
:
Advanced and low-cost 3D technologies (Laser Scanner and Digital Photogrammetry) are nowadays widely used in several fields such as Cultural Heritage and Archaeology. In this paper a collaborative project between the Archaeological Park of Herculaneum, the CISA (Interdepartmental Services Centre for Archaeology) and the DAAM (Department of Asia, Africa and Mediterranean Studies) of the University "L'Orientale" of Naples is presented. Aim of the project was to find a solution to study the Roman boat discovered in the ancient city of Herculaneum and destroyed by the eruption of Vesuvius in 79 A.D., without altering its state of preservation. Different digital survey techniques were employed to compare different types of sensors. The goal was to obtain an accurate definition of the construction characteristics of the boat thanks to the data fusion.
\end{abstract}

\section{INTRODUCTION}

\subsection{The Herculaneum boat}

In 1981 Giuseppe Maggi, at that time Director of the Herculaneum excavations, brought to light, along the South-West side of the Herculaneum, the ancient beach of the site, buried during the eruption of Vesuvius in $79 \mathrm{AD}$, and a series of arches below the sacred area of the town. The vaults, interpreted as a shelter of small boats or simply places for their maintenance, contained the skeletons of numerous people from Herculaneum who had looked for refuge there. On the 3rd of August 1982, on this ancient shore, exactly in front of the Suburban Thermae, an upturned boat was found (Camardo et alii, 2014).

The vessel had been buried by pyroclastic flows during the Vesuvius eruption and sealed in the blanket of volcanic materials that rapidly became harder so much that, with the lack of oxygen, the woods have been preserved through time. After uncovering the boat, a layer of silicone rubber was applied and on the external hull a fiberglass was used to create a shell in order to avoid the collapse of the charred structure of the boat remains. (Fig. 1) Once capsized and partially emptied of volcanic mud, in 1990 the boat was moved in the Antiquarium building in Herculaneum, under a protective covering.

The boat is nowadays stored in an air-conditioned exhibition pavilion carried out in 2009 in the Archaeological Park of Herculaneum. The vessel measures 7.8 meters, with a width of about 2.80 meters and a maximum height of approximately 1 meter from the keel to the gunwale.

The boat is sustained by a steel structure, made of two longitudinal axis and transversal beams following the shape of the hull (Pirozzi, 2000, fig. 8). This structure was created with the purpose of cushioning the seismic movements, keeping the boat elevated above the floor.

The exhibition hall is organized in a way that visitors can observe the boat from a ramp positioned along one end of the artefact. Survey operations were strongly affected by the arrangement of the exhibition hall and by the presence of the anti-seismic

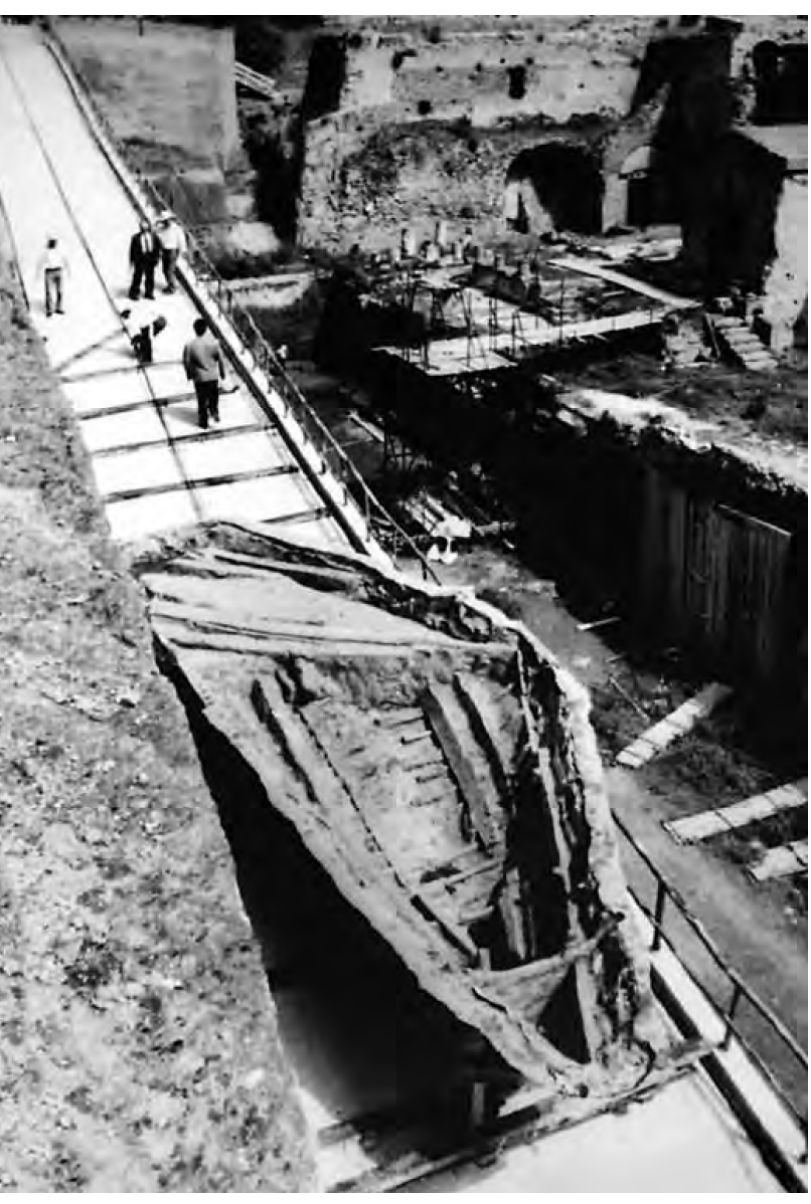

Figure 1. The carriage of the boat at the moment of discovering (from Camardo et alii, 2014)

\subsection{The restoration}


After the carriage of the boat, a first conservative work was to cover the wood with a layer of gauze and Japanese paper; this intervention aimed at stopping the carbonized wood crumbing and cracking as it progressively dried out and shrank. Once the boat was protected by the Japanese paper, the hull was then covered in a layer of silicon rubber about 1 centimetre thick. Finally, an external shell in fibreglass was created to contain the boat.

The boat was restored in 2008-2009 after an accurate multidisciplinary diagnostic campaign aimed at identifying the wood essence and at increasing knowledge about boating in Roman times. After the diagnostic and study phase, a series of samples were taken from various parts of the boat during its restoration in order to identify the wood used in its construction. A sample from the planking near the stem (sample A) proved to be pine (Pino $s p$ ), which is often used in carpentry and in particular for internal framework. Other two samples, (sample B and C) taken from the planking, were alder (Alnus glutinosa). This is a type of soft wood of the broad leaf tree family, which resists water well and is very compact. For this reason, it seems to have been used in the boat for the outer planking. Sample D, taken from the frame, was identified as beech (Fagus sylvatica). This is a hard wood of the broad leaf tree family. It is heavy, tends to split and warp, and is not very elastic; but it has the advantage that it can be bent with steam and the becomes harder and more flexible. In the boat it seems to have been used for the frames. The last sample (sample E) from the planking, showed that another type of wood was used, probably oak.

During the 2008 restoration works, performed under the supervision of ICR experts and carried out by an Italian restoration team, the fibreglass was partially cut off, the silicon rubber was removed and the protective gauze was taken away. The volcanic deposit partially filling the hull was then excavated and the sections of bent planking inside the boat were removed. The intervention was also addressed to delicately clean the wood, repositioning and reattaching fragments and filling in cracks. At the same time the metal nails were mechanically cleaned. When loose parts were put back in place, a suitably coloured fibreglass support was created.

The restauration work on the boat's interior planking was focused on finding the right strengthening treatments of air-dried archaeological waterlogged wood. The ICR team found a particular type of epoxy resin, used to consolidate the detached fragments and mixed with carbon powder, as plaster.

This work has allowed this exceptional archaeological find to be displayed. Nowadays, the boat is going to be restored again and the intervention could be the starting point of a working day on the conservation methods of carbonized wood and on the diagnostic techniques applicable to this type of cultural heritage.

\subsection{The first survey campaign}

A first photogrammetric survey was realized in 2015 by the CISA as part of the European Project "3D ICONS" with the major goal of digitalize some architectural monuments of the Herculaneum archaeological site and the boat. 3D-ICONS was intended to digitize a variety of archaeological and architectural European Heritage monuments in order to populate the digital library Europeana with 3D models and their metadata (D'Andrea, 2016). The 2015 photogrammetric survey has been obtained by using a

${ }^{1}$ The model is visible at the followed link:

https://www.europeana.eu/portal/it/record/2048703/object_HA 1780.html digital camera (Nikon D90) and a simple ladder for the shooting. 321 pictures were taken with a shooting distance of about 1 meter, obtaining a ground resolution of $0.3 \mathrm{~mm}$.

Through these parameters, with the use of Photoscan, a digital product of sufficient accuracy and medium resolution have been produced.

The model, actually shared online ${ }^{1}$, has been carried out with the aim to provide a light $3 \mathrm{D}$ replica easily manageable in remote way for different usages (tourism valorisation, education programmes, 3D printing for tactile fruition, etc.).

\subsection{The 2019 project}

Since the discovery of the boat in the 1980s, a complete and detailed study of the architectural characteristic of the Herculaneum boat has not been conducted yet by nautical archaeology specialists.

Richard Steffy of the Institute of Nautical Archaeology (Texas, US) visited the site of Herculaneum in 1982 and managed to conduct a short and preliminary documentation of the outer hull while still in situ and before the application of the fiberglass shell. His preliminary report remained unpublished but it is currently available online ${ }^{2}$; other general notes on the outer hull of the boat, issued on scientific publications, are the only detailed record we have so far (Steffy 1985, Steffy 1994, 67).

For this reason, that in 2019 the team of the University of Napoli "L'Orientale" decided to conduct a further photogrammetric survey of the Herculaneum boat with the aim to start a complete and systematic detailed study of the boat architecture ${ }^{3}$.

Currently, it is impossible to further document the outer hull, initially observed by Steffy, because it is partially hidden by the fiberglass shell, while the inner hull is visible and available for the study. Despite that, the exhibition arrangement of the boat and its current state of preservation does not allow researchers to approach closely the inner hull. Therefore, it is difficult to take measurements, to observe in detail the remains, and to collect useful data to properly analyse the construction of the boat.

Thus, the team decided to experiment a high-resolution photogrammetric survey of the boat which would allow to measure and describe it in detail. The other aim of the project is to use the 3D model resulting from the photogrammetry in order to model each components of the boat carpentry (longitudinal and transversal) and the junctions (pegs, mortise and tenons, nails and treenails). This work will allow to conduct further analysis which could lead to the hypothetical reconstruction of the original boat. Therefore, it was a priority, in this first step of the project, to obtain a final digital model at the highest possible resolution.

\section{METHODOLOGY}

\subsection{The choice of the sensors}

In order to gain a geometrically and chromatically higher resolution reproduction of the inner boat, adequate also to detect the exact position of every pegs, treenails and nails, and the longitudinal and transversal carpentry, a new survey field season has been conducted in June and July 2019.

Different digital survey techniques were employed, taking advantage of the possibility to test different types of sensors and to integrate the data, in order to understand the potentiality of

2

https: \www.academia.edu/12869484/2005. The Herculaneum Boat_Project._Unpublished_manuscript

${ }^{\overline{3}}$ The study of architectural characteristics of the boat will be conducted in collaboration with the Centre Camille Jullian CNRS (Aix-en-Provence). 
their fusion.

To facilitate the overlapping of the different acquired datasets, the first step was to create a topographic network by using a Total Station. Six targets have been appointed on the external support of the boat and then acquired.

The pavilion has a structure made of big windows that guarantee a natural light source; nevertheless, these light spots are concentrated in the area of the bow of the ship, even because the upper openings are covered with curtains; this situation creates an uneven spread of light inside the structure.

To overcome the obstacles of acquisition, due to the structure supporting the boat, a photogrammetric survey, using two different sensors mounted on a telescopic bar, was planned; a full frame digital camera (Nikon D750) and a DJI OSMO system (20MPX) were used. While the professional camera assures a detailed acquisition, the latter has the advantage of being extremely versatile and light, as well as functional to extreme acquisitions, thanks to the built-in gimbal which guarantees stability to the shots.

The Close-Range photogrammetry survey was followed by imaging laser scanning with a Leica BLK360, connected up with the ReCap Pro software for the acquisition and elaboration of the point cloud. The ortho-photos obtained from the processing of the acquired data allow setting up a CAD characterization of the inner of the boat.

Given the complexity of acquisition, due to the position of the boat and to the presence of the supporting structure of the object, a further data-capture has been performed with a thermal-camera adopting the same acquisition system chosen for the DJI OSMO system.

\subsection{Close-Range photogrammetry survey}

As we have seen, the detailed requirements of the 2019 project made the 2015 survey insufficient.

If, on one side, the use of the Nikon D750 full frame reflex camera guarantees high-resolution images, on the other side, its size and weight make difficult to capture stable images by mounting the camera on a telescopic pole. Although it is possible to active the shot remote control, thanks to the wi-fi connection, the limited light, which means lower shot, increases the risk of blurring for the photos, as it is not recommended for this photogrammetric technique to choose a lower diaphragm.

A first acquisition's swipe has been carried out by sliding the telescopic pole with the Nikon around the boat to record the internal lateral axles and the ribs. Furthermore, to better acquire the inside of the hull, the camera has been mounded zenithally under a bar that ran for the entire length leaned on the iron beams supporting the boat (Fig. 2).

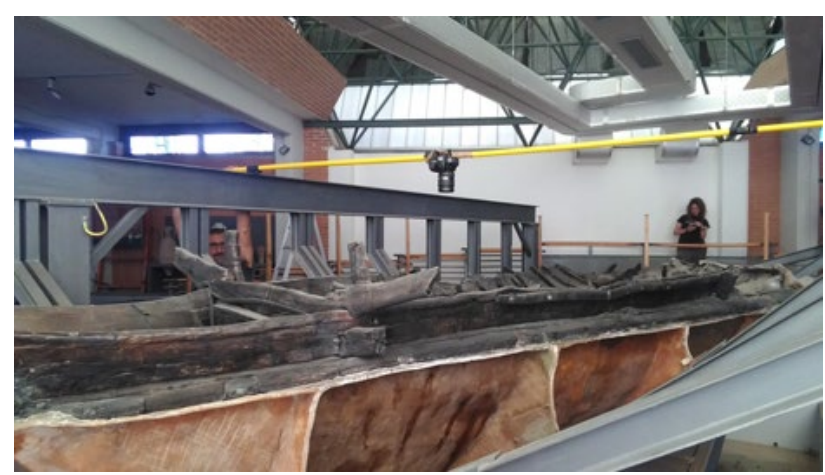

Figure 2. Digital camera (Nikon D750) bounded under a telescopic bar for zenithal photogrammetric survey.
The choice of the two different techniques modes was due to the necessity of minimizing the resolution deviation caused by the different shooting cone (Fig. 3).

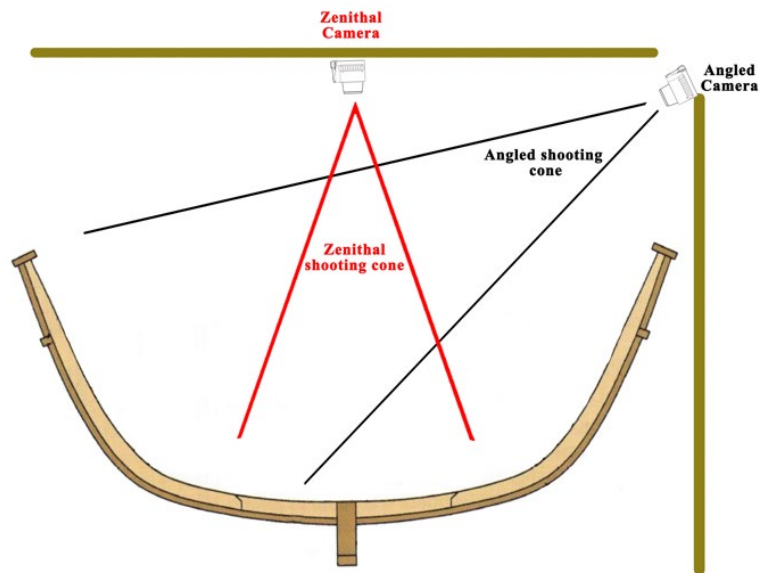

Figure 3. Simplified scheme of the shooting cones for the different acquisition methods.

While the angle of shot of the camera, positioned on telescopic pole moved along the external side of the boat, is oblique with planes at different scale, the zenithal acquisition reduces the field of view to the "normals". This last technique acquires a sequence of images where the extraction of tie points is a simple task for the lack of convergent images or scale variations (Barazzetti 2017).

The integration of these two data-acquisition methods produced a final model with a more homogeneous resolution thanks to the removing of the less focus parts belonging to the dataset extracted from the angled images; in the post-processing phase the images were masked to hide the blur parts.

In short, about 262 photos have been acquired and processed according to a well-proven workflow in Metashape (Fig. 4). The alignment (set to medium quality) generated a dense point cloud of about 26 million points, a model of about 57 million faces and a medium resolution of $0.32 \mathrm{~mm} /$ pixel.

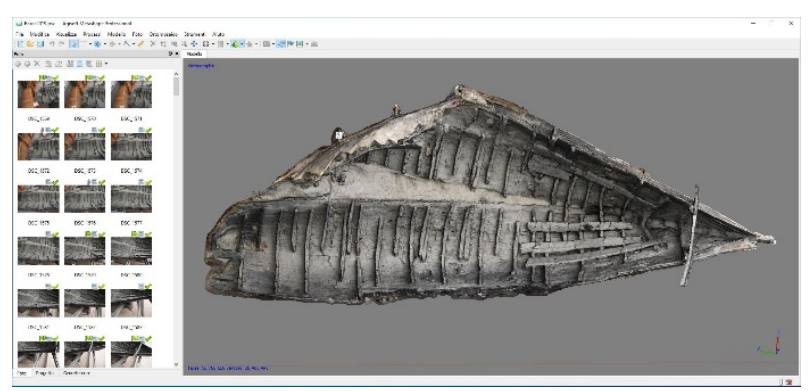

Figure 4. Screenshot of the mesh obtained from the photogrammetric acquisition (Elaboration with Agisoft Metashape Professional).

During the same day, another photogrammetric survey has been carried out by using DJI OSMO system with a Zenmuse X5S sensor of 20.8 MPX. Also, this camera has been mounted on the telescopic pole, with the advantage of having a highly optimized 3 -axis gimbal with pinpoint-precise motor rotation. The remote control of the shots was done through the DJI app installed on the tablet, which controls also the movement of the axis of the camera. This shot technique allows to have a strong stability of the camera and to acquire quickly many photos avoiding the risk 
of capturing blurred pictures. The data-acquisition has been performed moving the pole around the boat; 240 photos were taken. The alignment (set to medium quality) generated a dense point cloud of about 4 million points, a model of about 10 million faces and a medium resolution of $0.80 \mathrm{~mm} /$ pixel.

From both final models, two top-view ortho-photos were extracted and compared visually. Notwithstanding the high resolution (both were under $1 \mathrm{~mm}$.), the definition of the details results greater in the Nikon acquisition; this target has been reached after an accurate data-acquisition phase (angular and zenithal) and a long post-processing work (Fig. 5).

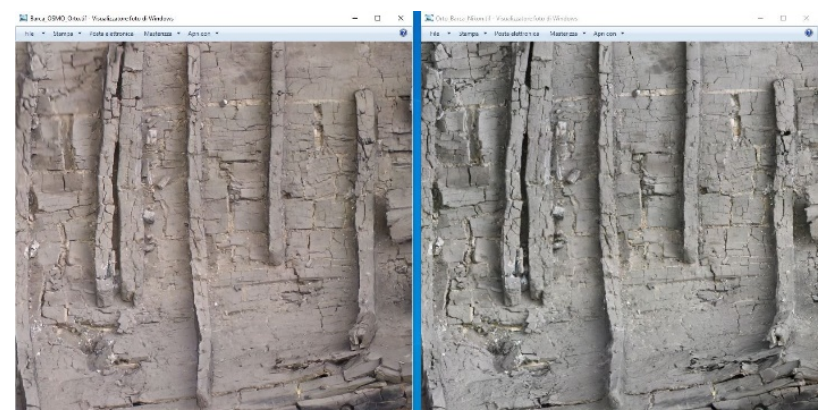

Figure 5. A visual comparison of the ortho-photos extracted by the close-range photogrammetry data. On left the OSMO acquisition and on the right the Nikon one.

\subsection{Laser scanning}

Even if the model generated by the photogrammetric survey had given satisfactory results, the presence of the darker and deeper areas didn't ensure a precise geometric restitution. In order to improve and increase the geometrical accuracy of the digital replica of the boat, a successive acquisition campaign was carried out with a laser scanner.

Data-acquisition with laser scanner isn't affected by light conditions and the geometric accuracy is unvaried if the distance of the instrument to the object is fixed. For this aim, a Leica BLK360 was chosen; the instrument is ultra-compact, light and, mainly, can be hooked upside-down. Furthermore, the laser scanner acquires around $360.000 \mathrm{pts} / \mathrm{sec}$ and produces for each scan a spherical image of 150MPX.

According to the Nikon zenithal acquisition, the laser scanner was mounted on the horizontal bar which has been moved along the iron beams supporting the boat (Fig. 6).

At the end, 12 scans were taken, each one of 11-12 million points, which were aligned during the data-acquisition phase with the Recap Pro software installed on the tablet. Considering the wide range of acquisition of the laser scanner $(\max 60 \mathrm{~m})$, the final restitution included not only the boat, but even the whole pavilion.

The complete model, composed originally by 143 million of points, was cropped removing the parts of the pavilion; at the end only the 17 million of the boat were stored.

\subsection{Thermal-camera application}

Because of the supporting structure obstacles and difficulties in

\footnotetext{
${ }^{4}$ Whereas the measured radiation is influenced by the external environment, every thermal analysis should be conducted with the camera setting of the temperatures, of the value of the material emissivity and of the distance from the investigated object.

${ }^{5}$ The camera was provided by the Analist Group srl.
}

closely examining the boat, it was decided to integrate the $3 \mathrm{D}$ models, generated from the photogrammetric and scanner acquisition, with a thermal analysis; the survey has benne carried out in order to detect the position of potential metal nails not well visible in the charred planking and, furthermore, in an attempt to acquire knowledge about the preservation state of the boat.

Thermography is a technology that allows to acquire a thermal map of the surface of an object detecting the IR radiation emitted from the material. Infrared imaging is widely used in archaeology and heritage diagnostics allowing non-invasive mapping of many features, at surface and subsurface levels, in order to obtain important information about diagnostics at the basis of any scientific conservation and valorisation work (Maldague, 2001). Through a thermal camera the technique turns the emission pattern of an object into a visible image.

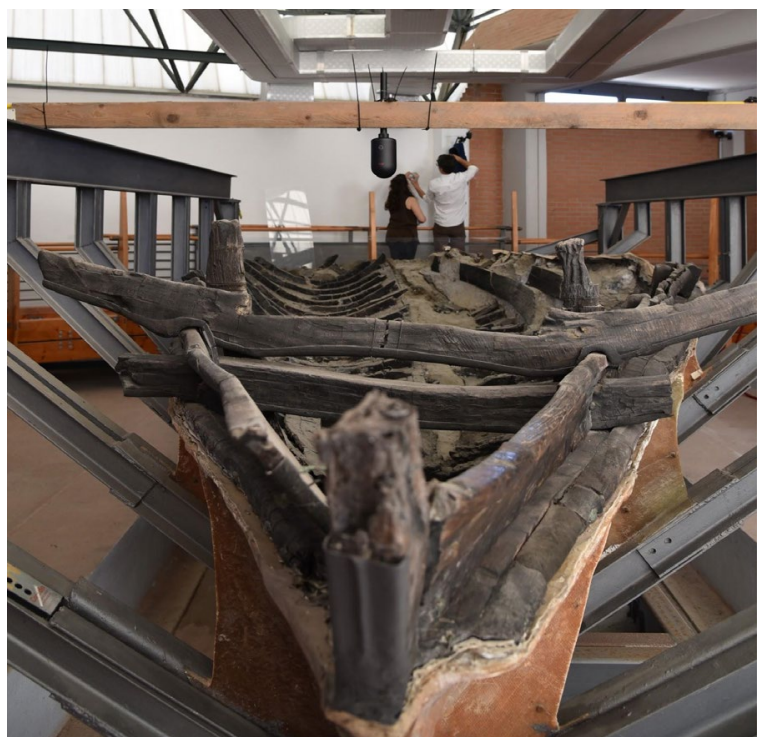

Figure 6. The laser scanner BLK360 mounted, with the help of wooden ribs, on a pole above the boat.

Thermographic techniques are aimed at recognizing hidden structural elements (in the soil or in architectural buildings), lesions and detachments of frescoes or wall painting, stagnation of humidity in masonry and plaster.

In this project other materials, such as carbonized wood and metal (of the nails), have been considered analysed. Although the infrared thermography, as a non-destructive testing (NDT), has been often applied to different type of material, its application on archaeological carbonised wood is not well documented in the literature ${ }^{4}$.

In this occasion a thermal camera Flir Tau2 640 (FPA / Digital Video Display Format: 640x512), with a spectral range of 7,5 $13 \mu \mathrm{m}$, was used ${ }^{5}$. An in situ passive thermography method ${ }^{6}$ was applied on the boat.

The camera was mounted on a pole and moved by an operator around the perimeter of the object at a distance of about $1.5 \mathrm{~m}$ from the boat's hull. The sensor was connected to a hand pad which enables thermal data recording and visualisation in real

6 Passive Thermography directly measures the surface temperature evaluation, since the examined region will have abnormal hot-spots compared with the surroundings. The active methodology, instead, measures the surface temperature after the generation of an excitation such that the external heat source produces a thermal contrast between the feature or object of interest and the background. 
time (Fig. 7).

The final tiff image, exported from the acquisition (with a resolution of $2184 \times 2257 \mathrm{pixel} / \mathrm{cm}$ ), has been analysed in Analist Cloud 2019 software. The image has been overlapped and aligned with the top-view ortho-photo extracted by the photogrammetry carried out with the Nikon.

The processed thermal image includes also the steel support structure and part of the pavilion; thus, the survey has produced an IR Histogram with a temperature range between 22 and $31.8^{\circ} \mathrm{C}$ (Fig. 8).

As for the planking, no temperature differences indicating the presence of heterogeneous materials, such as metal nails or pins, were detected, with the exception of a modern copper coin (2 cents of $€$ ) which was accidentally at the bottom of the boat (Fig. $9)$. The difference of temperature between the coin and the rest of the carbonised hull is about $1.6^{\circ} \mathrm{C}$.

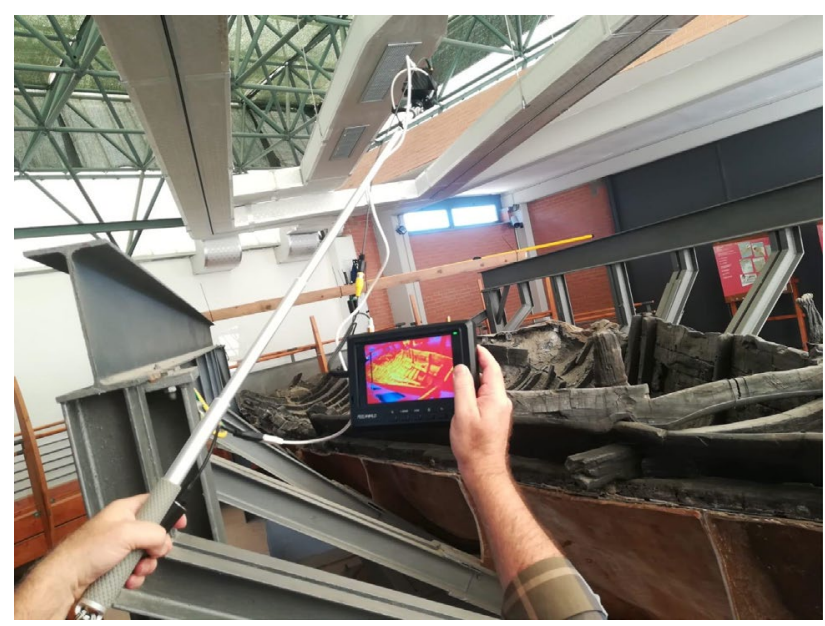

Figure 7. Thermal data-acquisition

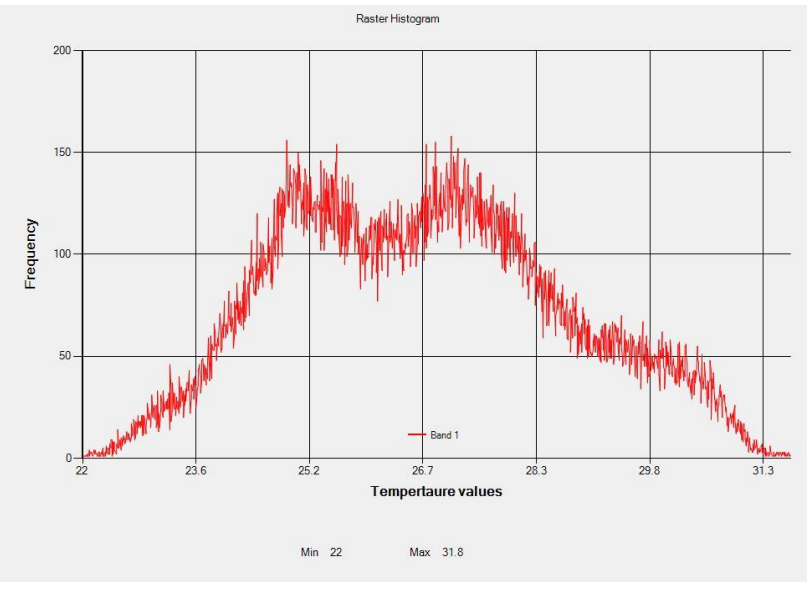

Figure 8. The Raster IR Histogram

The fact that the recent coin stands out so clearly in the thermal image, unlike the ancient nails, suggests that the insulating properties of protective films, used in the restoration project, impede relevant alterations in emissivity ${ }^{7}$.

Some shadows visible in the thermal image have been recognized thanks to the overlapping with the ortho-photo; these colder features can be identified as nails.

${ }^{7}$ It needs to be also considered the fact that the thermal survey was conducted without knowing the exact temperature of the pavilion (cooled with air conditioning) or the emissivity value of
With the exception of these detailed "anomalies", no further data can be highlighted. A possible solution for a more complete documentation could be the utilisation of an active methodology requiring an external stimulus which warms the boat; in this way it is possible not only to determine the temperature of surfaces, but also to calculate the thermic emission by repeating the measurements in a chronological interval of few minutes. The sequential monitoring of the surface could produce a complete set of qualitative and quantitative information related to different materials and features of the boat (Theodorakeas et alii, 2015).

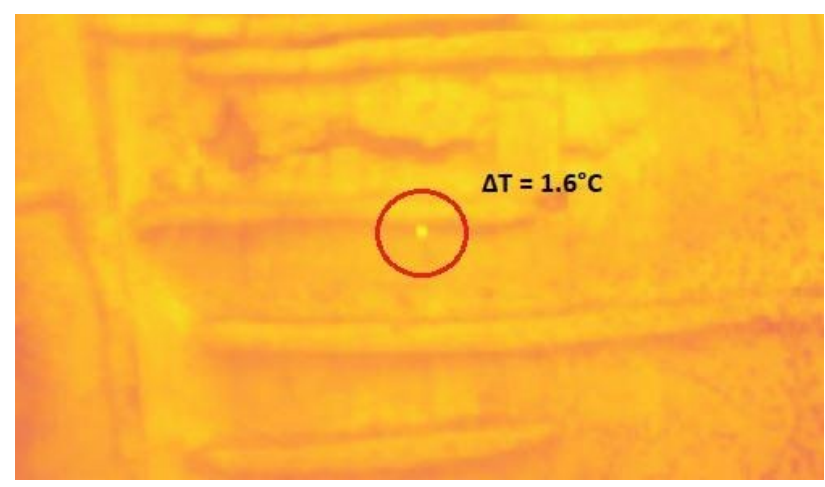

Figure 9. Detail of the thermal image with the modern coin placed on the planking

Despite these limitations, some interesting results can be already pointed out. If on the one hand the trace of the nails stuck in the boards was not detected, on the other hand, few anomalies were found in some areas of the boat. From the overlapping with the ortho-photo, the thermal image shows some heat spots on the surface of the hull, toward the bow, in correspondence of the dunnage planks. On the left side of the hull near to this anomaly the sensor registered a colder temperature of the boat, probable linked to the humidity stagnation in the curved profile of the side wall (Fig. 10).

The nature of these thermal anomalies should be better analysed in future; at this stage, these differences in heat could represent an area where the air conditioning of the room mainly influences the IR radiation of the surfaces. In this context the analysis can be considered a first attempt for the development of an appropriate conservation monitoring of the boat.

\section{RESULTS AND FUTURE PROSPECTIVE}

The paper addressed to highlight the integration of different 3D range and image-based techniques with diagnostic analysis. The project aims at developing a particular methodological approach in problematic survey condition.

Documenting an archaeological artefact is a hard task, since the manufact often is fragmentary or fragile and sometime is restored and protected in showcases. Many archaeological objects cannot be drawn according to the best documenting techniques and for these motives it is necessary to develop an alternative strategy. In this project the lights, the air-conditioning, the pavilion and the ramp represented factors which impeded a good digital survey. The first 3D replica of the boat, carried out in 2015, was not so detailed for the aim of an in-depth analysis. Different reasons pushed to define a new approach based on the data fusion of distinct dataset acquired by various sensors and with different shooting techniques.

the different types of wood. 
The Nikon acquisition produced a detailed model with 26 million points with a resolution less than $1 \mathrm{~mm}$. The camera acquired photos oblique and zenithal. The OSMO rendered a final model of 4 million points with a resolution lower than Nikon, but always less than $1 \mathrm{~mm}$. Finally, a laser scanner acquisition was carried out to increase the accuracy of the geometry.

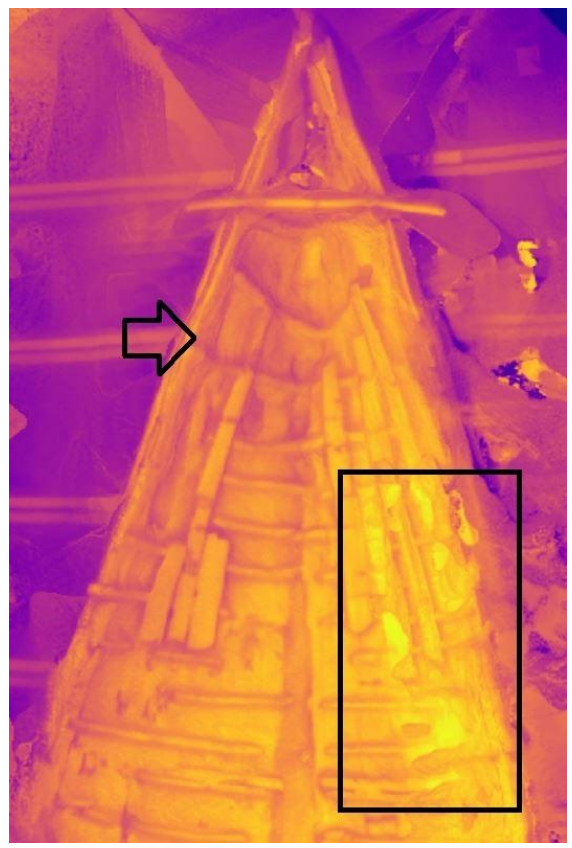

Figure 10. Anomalies of temperature near the bow (in the square the yellow spots are the hottest areas; the arrow indicates the colder areas)

The datasets were aligned and fine registered in CloudCompare with the model obtained by the Nikon as reference; the RMS is between 0.022 and $0.025 \mathrm{~m}$. The distance among the point-clouds calculate by CloudCompare shows an average distance of 0.003 $\mathrm{m}$. All the three datasets were merged and sampled by removing the duplicate points at a distance greater than $0.001 \mathrm{~m}$. The final model is 31 million points larger than the Nikon one.

Many recent researches (Nocerino et alii 2014, Barazetti 2017) focused on the geometric resolution and the way to check the accuracy of the 3D dataset highlighting the potentiality of both data-acquisition techniques (close-range photogrammetry and laser scanner) to render final precise models. Another parameter to be checked is the reliability intended as the precision of the image coordinates. Notwithstanding, the comparison between the two ortho-photos by Nikon and OSMO, shown at Fig. 5, and the differences between the two meshes (Fig. 11) proves that the geometrical correctness of the models not always provides good results.

All generated models have a high-resolution and, from a spatial point of view, are reliable. But the visual analysis shows that the sensor is important in terms of clearness and sharpness of the profiles of the single architectural component of the boat.

The ortho-photo and mesh generated by Nikon registered details with better definition. As the results will be used to understand and draw the particulars of the boat, only a combination between the photos and the subsequent processing (ortho-photos, pointclouds and meshes) can assure an optimal graphical render of the archaeological artefact.

From the three merged datasets a mesh has been carried out with 60 million faces. In order to exploit the richness of the data fusion process we planned to re-texturized the model with the orthophoto generated by Nikon through an optimisation of the mapping process (Inzerillo et alii 2019). We expect to improve the visual inspection of the architectural component of the boat and make possible a correct 3D modelling of the object. Furthermore, the high-quality of the model could be used by different users and for various needs.

Finally, the digital survey could better support a new restoration intervention providing more and detailed information about the state of the conservation and, mainly, the technical plan of the boat; furthermore, a new thermal acquisition, carried out by an non-invasive active methodology, could support the analysis giving more data about the different materials used to build the boat.

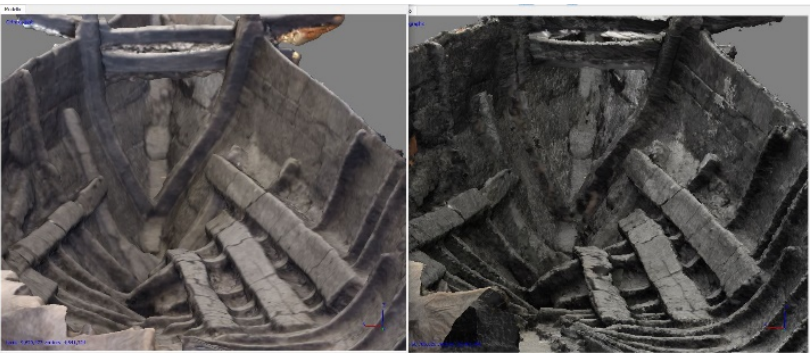

Figure 11. The comparison between the meshes from OSMO (on left) and Nikon (right): the boards of the floor and the ribs in the model generated by OSMO are more rounded and smoothed.

\section{ACKNOWLEDGEMENTS}

The authors would like to express their gratitude to Francesco Sirano, Director of the Archaeological Park of Herculeanum, for having authorized and supported the project and Antonio Iannuzzi, CEO of the Analist Group srl, for his suggestions and assistance in laser scanner and thermal acquisition.

\section{REFERENCES}

Barazzetti, L., 2017. Network Design in Close-Range Photogrammetry with Short Baseline Images, ISPRS Annals of the Photogrammetry, Remote Sensing and Spatial Information Sciences, Volume IV-2/W2, 17-23

Camardo, D., Court, S., Guidobaldi, M. P., Notomista, M., 2014. Ercolano e il mare. Archeo. Attualità del passato, 354.

D'Andrea. A., 2016. Providing 3D Content to Europeana. CAA 2015. Keep the Revolution Going. Proceedings of the 43rd annual Conference on Computer Applications and Quantitative Methods in Archaeology, 1, 269-274.

Inzerillo L., Di Paola F., Alogna y., 2019. High-quality texture mapping process aimed at the optimisation of 3D structures light models. The Int. Arch. Photogramm. Remote Sens. Spat. Inf. Sci., Volume XLII-2/W9, 389-396.

Pirozzi, M. E., 2000. La barca romana di Ercolano. Spazio immagine e forma di un progetto di musealizzazione. Rivista di Studi Pompeiani, 11, 281-284.

Maldague, X. P. V., 2001. Theory and Practice of Infrared Technology for non-Destructive Testing. Wiley, New York.

Nocerino, E., Menna, F., Remondino F., 2014. Accuracy of typical photogrammetric networks in cultural heritage $3 \mathrm{D}$ 
modelling projects. The Int. Arch. Photogramm. Remote Sens. Spat. Inf. Sci., Volume XL-5, 465-472.

Steffy, J. R., 1985. The Herculaneum Boat: Preliminary Notes on Hull Details. American Journal of Archaeology, 89.3, 519-21.

Steffy J. R., 1994. Wooden Ship Building and the Interpretation of Shipwrecks. College Station: Texas.

Theodorakeas, P., Cheilakou, E., Ftikou, E., Koui, M., 2015. Passive and active infrared thermography: An overview of applications for the inspection of mosaic structures. Journal of Physics: Conference Series, 655. 